\title{
Polydatin Protects Bovine Mammary Epithelial Cells against Zearalenone-Induced Apoptosis by Inhibiting Oxidative Responses and Endoplasmic Reticulum Stress
}

\author{
Yurong Fu ${ }^{1,+}$, Yongcheng Jin ${ }^{1,+}+{ }^{+}$, Anshan Shan ${ }^{2}$, Jing Zhang ${ }^{1, *}$, Hongyu Tang ${ }^{1}$, Jinglin Shen ${ }^{1}$, Changhai Zhou ${ }^{1}$, \\ Hao Yu ${ }^{1}{ }^{1}$, Hengtong Fang ${ }^{1}$, Yun Zhao ${ }^{1}$, Junxiong Wang ${ }^{1}$ and Yue Tian ${ }^{1}$ \\ 1 Key Laboratory of Zoonosis Research, Department of Animal Science, College of Animal Sciences, \\ Jilin University, ministry of Education, Changchun 130062, China; fuyr20@mails.jlu.edu.cn (Y.F.); \\ ycjin78@163.com (Y.J.); tanghy@jlu.edu.cn (H.T.); shenjinglinshen@aliyun.com (J.S.); zhouch@jlu.edu.cn (C.Z.); \\ yu_hao@jlu.edu.cn (H.Y.); fanght@jlu.edu.cn (H.F.); zhao_yun@jlu.edu.cn (Y.Z.); \\ jxwang19@mails.jlu.edu.cn (J.W.); letian20@mails.jlu.edu.cn (Y.T.) \\ 2 Institute of Animal Nutrition, Northeast Agricultural University, Harbin 150030, China; asshan@neau.edu.cn \\ * Correspondence: zhang_jing99@jlu.edu.cn; Tel.: +86-147-9436-7799 \\ + YuRong Fu and Yongcheng Jin should be considered joint first author.
}

Citation: Fu, Y.; Jin, Y.; Shan, A.; Zhang, J.; Tang, H.; Shen, J.; Zhou, C.; Yu, H.; Fang, H.; Zhao, Y.; et al.

Polydatin Protects Bovine Mammary Epithelial Cells against Zearalenone-Induced Apoptosis by Inhibiting Oxidative Responses and Endoplasmic Reticulum Stress. Toxins 2021, 13, 121. https://doi.org/ $10.3390 /$ toxins 13020121

Received: 5 January 2021

Accepted: 2 February 2021

Published: 5 February 2021

Publisher's Note: MDPI stays neutral with regard to jurisdictional claims in published maps and institutional affiliations.

Copyright: (c) 2021 by the authors. Licensee MDPI, Basel, Switzerland. This article is an open access article distributed under the terms and conditions of the Creative Commons Attribution (CC BY) license (https:/ / creativecommons.org/licenses/by/ $4.0 /)$

\begin{abstract}
Zearalenone (ZEA) is a mycotoxin of the Fusarium genus that can cause endoplasmic reticulum (ER) stress and Apoptosis in bovine mammary epithelial cells (MAC-T). Polydatin (PD), a glycoside purified from Polygonum cuspidatum, has antioxidant properties. This study aimed to explore whether PD can alleviate ZEA-induced damage on bovine mammary epithelial cells (MAC-T). We found that incasing the concentration of ZEA $(0,7.5,15,30,60,90,120$, and $240 \mu \mathrm{M})$ gradually decreased the cell viability. PD treatment alone at 5, 10, and $20 \mu \mathrm{M}$ did not affect cell viability. Followup studies then applied $30 \mu \mathrm{M}$ of ZEA and $5 \mu \mathrm{M}$ of PD to treat cells; the results showed that the ZEA + PD treatment group effectively reduced cell oxidative damage compared with the ZEA treatment group. The qPCR analysis showed that ZEA treatment significantly up-regulated the expression of ER stress-related genes, relative to the control. However, adding PD significantly down-regulated the expression of ER stress-related genes. The cell apoptosis detection results showed that, compared with the ZEA treatment group, the ZEA + PD treatment group down-regulated the Bax gene and up-regulated the $B c l-2$ gene expressions, which reduced the cell apoptosis rate and Caspase-3 activity. Taken together, these results indicate that PD reduces ZEA-induced apoptosis by inhibiting oxidative damage and ER stress.
\end{abstract}

Keywords: apoptosis; bovine mammary epithelial cells; endoplasmic reticulum stress; polydatin; zearalenone

Key Contribution: ZEA induces oxidative damage to bovine mammary epithelial cells, leading to various effects. PD can alleviate the oxidative stress response of bovine mammary epithelial cells to ZEA by reducing the subsequent ER stress response.

\section{Introduction}

Unsuitable storage conditions and variable temperatures can promote the production of mycotoxins in harvested crops. Zearalenone (ZEA) is a fusarium toxin commonly found in feed products. Zearalenone is cytotoxic [1] to the liver, spleen [2], intestines [3], and hematopoietic cells [4]. It induces the production of reactive oxygen species (ROS) and triggers intracellular oxidative stress, which has subsequent cytotoxic and genotoxic effects [5]. Furthermore, oxidative damage reduces the performance of dairy cows and affects their disease resistance, which reduces milk yield and quality [6]. Oxidative stress is closely related to the cow's physiology and nutritional status factors. Excessive ROS production leads to oxidative stress, loss of cellular function, and, ultimately, apoptosis 
or necrosis $[7,8]$. Our previous studies have shown that adding ZEA to bovine mammary epithelial cells promotes excessive ROS generation, endoplasmic reticulum (ER) stress, and apoptosis [9]. The balance between reduced glutathione (GSH) and oxidized glutathione (GSSH) is essential for maintaining cellular redox homeostasis. However, GSH gets depleted during protein misfolding, resulting in ROS generation [10]. Various parameters, such as malondialdehyde (MDA), reduced glutathione (GSH), total superoxide dismutase (T-SOD), and antioxidant enzyme activity, among others, are related to oxidative stress, and can indicate the cellular levels of oxidative stress [11].

ZEA was previously detected in milk samples. Therefore, whether ZEA may have similar effects on bovine mammary epithelial cells is worth studying. Studies have shown that ZEA induces mouse Leydig cell apoptosis by activating the ER stress-dependent signaling pathway [12]. The function of the ER is sensitive to the accumulation of unfolded proteins, calcium homeostasis, and redox state changes; thus, disruption of these processes can cause ER stress. Since the unfolded protein response (UPR) is an ER stress response that maintains cell homeostasis, deregulation of UPR can cause cell disorders and cell death [13]. However, previous studies found that ZEA can induce apoptosis of MAC-T cells. Mammalian cells express the UPR transducer proteins IRE1, PERK, and ATF6, which control transcriptional and translational responses to ER stress [14]. Under non-stress or physiological conditions, these proteins remain in an inactive state, and bind to the molecular chaperone BiP/GRP78, which is also the primary regulator of ER stress. Under ER stress, GRP78 decomposes from the stress sensors, thus activating them. Once activated, PERK phosphorylates the $\alpha$ subunit of the translation initiation factor eIF2 $\alpha$ (eukaryotic initiation factor $2 \alpha$ ). This activation results in an overall reduction of translation, and promotes priority translation of UPR-dependent genes (such as activated transcription factor 4 (ATF4)). The vital target of ATF4 is CHOP (C/EBP homologous protein) [15].

Oxidative stress and ROS generation are indispensable components of UPR. Therefore, ROS generation can occur both upstream and downstream of UPR. Both oxidative and ER stresses are involved in various physiological and pathophysiological conditions, and they play vital roles in cell homeostasis and apoptosis. This fact suggests that ER stress and oxidative stress have a significant correlation. For instance, our previous studies have shown that ZEA can cause ER stress and apoptosis in MAC-T cells. However, whether there are compounds that can alleviate the effects of ZEA remain unclear.

Polydatin (3,4',5-trihydroxystibene-3- $\beta$-mono-D-glucoside; $\mathrm{PD})$ is a natural precursor of resveratrol and an active ingredient in various Chinese medicines, such as Polygonum cuspidatum. Polydatin is the product of resveratrol and glucose, hence, it is also called resveratrol glycoside. Its pharmacological effects are similar to resveratrol, and they can be transformed into each other in the body. However, PD has a stronger antioxidant effect and stability [16]. PD may also exist in peanuts, grapes, and wine [17]. It has a wide range of positive health effects, including antioxidant [18], anti-cardiovascular diseases [19], anti-inflammatory [20], and inhibition of cancer cell growth [16], and can be used for the treatment of shock [21]. Polydatin can protect bone marrow stem cells from oxidative damage [22]; however, it is unclear whether PD can rescue cells from ER stress and apoptosis. Here, we investigated whether PD protects bovine mammary epithelial cells from ZEA-induced ER stress and apoptosis. In this study, various in vitro experiments were conducted to determine whether the PD-related effect caused by ZEA on MAC-T cells can be alleviated.

\section{Results}

\subsection{The Increase in PD Decreased the MAC-T Cell Viability Due to ZEA}

Cell viability was measured by the CCK- 8 assay. We found that ZEA exposure significantly reduced the MAC-T cell viability, and viability decreased with increasing ZEA concentrations (Figure 1a). Cells exposed to $30 \mu \mathrm{M}$ of ZEA had significantly lower survival than the control group $(p<0.001)$. Therefore, cells were treated with $30 \mu \mathrm{M}$ of ZEA in subsequent experiments. MAC-T cells treated with 5-20 $\mu \mathrm{M}$ of PD had similar survival 
rates as the control group, suggesting that PD does not affect cell survival (Figure 1b). Given that all concentrations yielded similar results, $5 \mu \mathrm{M}$ of PD was used for subsequent experiments. Interestingly, MAC-T cells exposed to ZEA + PD had significantly higher survival rates than cells treated with ZEA alone $(p<0.01$; Figure $1 c)$. The cell viability of the ZEA treatment group decreased by about $13 \%$ relative to the control group. Compared with the ZEA treatment group, the ZEA + PD treatment group increased by about 9\%. The addition of PD effectively increased cell viability due to ZEA inhibition.

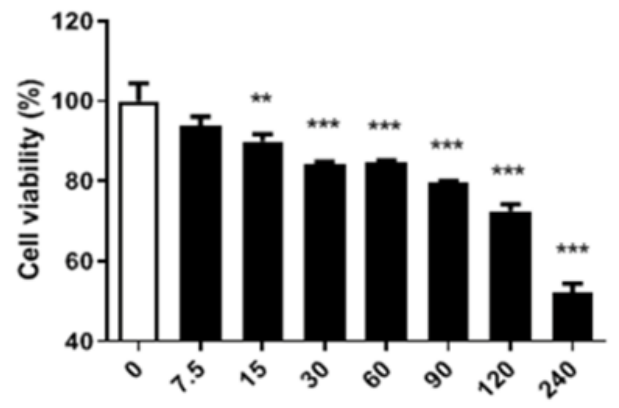

(a)

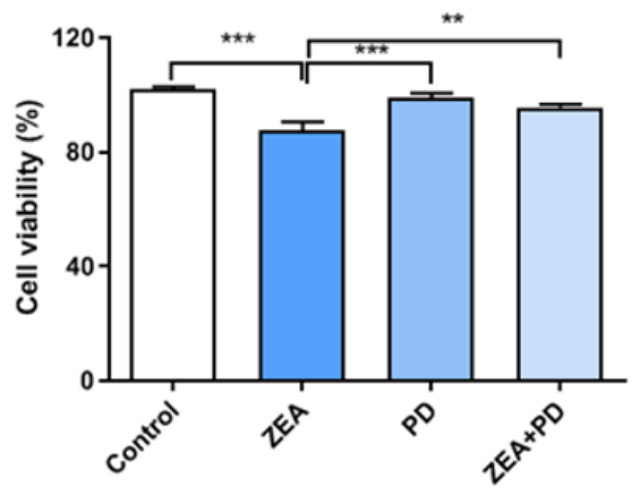

(c)

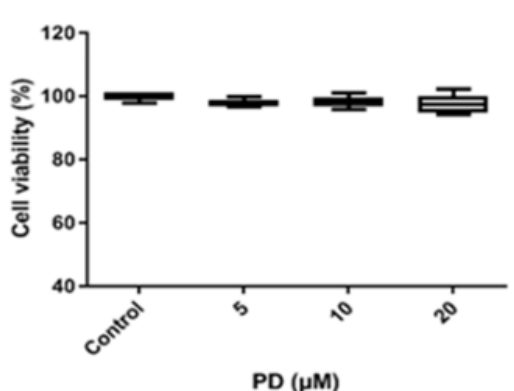

(b)

Figure 1. Effects of Zearalenone (ZEA) and Polydatin (PD) on the viability of bovine mammary epithelial cells (MAC-T). (a) Viability of MAC-T cells treated with different concentrations (0, 7.5, $15,30,60,90,120$, and $240 \mu \mathrm{M}$ ) of ZEA for $24 \mathrm{~h}$. (b) Viability of MAC-T cells treated with different concentrations $(0,5,10$, and $20 \mu \mathrm{M})$ of PD for $24 \mathrm{~h}$. (c) Effects of $30 \mu \mathrm{M}$ of ZEA and $5 \mu \mathrm{M}$ of PD on MAC-T cell viability. Figure 1c shows the control group, ZEA treatment group, PD treatment group, and ZEA + PD treatment group. Each experiment was repeated three times. All values are expressed as mean $\pm \operatorname{SEM}(n=3)$. In the figure, ${ }^{* *} p<0.01,{ }^{* * *} p<0.001$.

\subsection{PD Reduces the ZEA-Induced Cytotoxic Effect in MAC-T Cells}

The lactate dehydrogenase (LDH) activity, measured by quantifying the LDH levels released from disrupted cells into the culture media, can serve as a proxy for cytotoxicity. The results showed that LDH increased with increasing ZEA concentrations (Figure 2a). Compared with the ZEA-treated group, the LDH content of cells treated with ZEA + PD was significantly reduced (Figure $2 b, p<0.001$ ). Meanwhile, the LDH content of the ZEA treatment group increased by about $43 \%$ relative to the control group. Compared with the ZEA treatment group, the ZEA + PD treatment group decreased by about $23 \%$. These results suggest that PD lessens the cytotoxic effect in MAC-T cells treated with ZEA due to ZEA and PD interactions. 


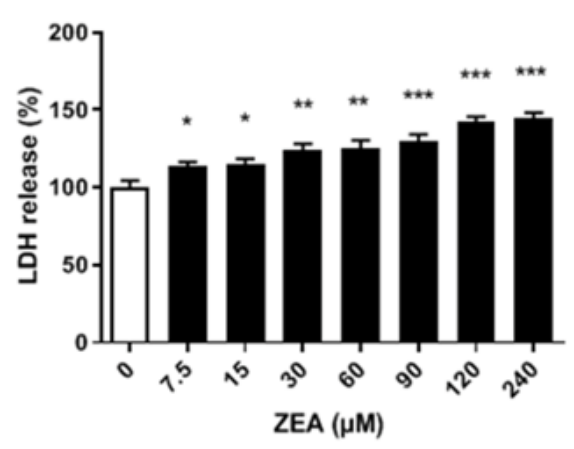

(a)

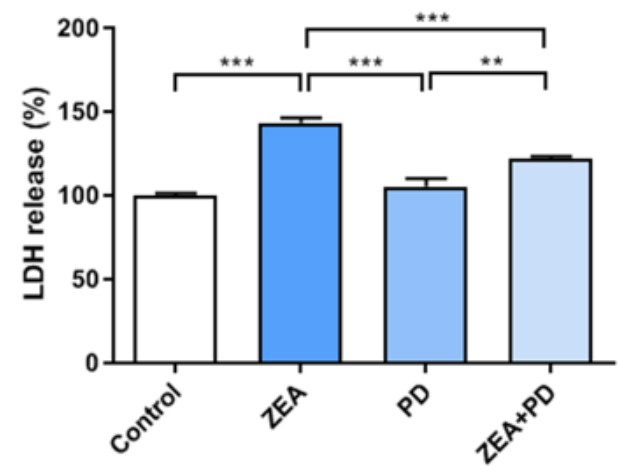

(b)

Figure 2. Effects of ZEA and PD on the lactose dehydrogenase (LDH) levels of MAC-T cells. (a) LDH released by MAC-T cells treated with varying ZEA concentrations $(0,7.5,15,30,60,90,120$, and $240 \mu \mathrm{M}$ ) for $24 \mathrm{~h}$. (b) LDH released by MAC-T cells exposed to $30 \mu \mathrm{M}$ of ZEA and $5 \mu \mathrm{M}$ of PD. In the figure, ${ }^{*} p<0.05,{ }^{* *} p<0.01,{ }^{* *} p<0.001$.

\subsection{PD Can Inhibit ZEA-Induced Oxidative Damage}

Previous results indicate that ZEA and PD can increase cell viability and reduce the LDH activity of treated cells. Therefore, whether the effect of oxidative damage is obvious requires further investigation. This study showed that the MDA level of the ZEA treatment group was significantly higher than that of the control group $(p<0.01$, Figure 3a). In contrast, the MDA level of the ZEA + PD group was significantly lower than the ZEA group $(p<0.001)$. Compared with the control group, the MDA level of the ZEA group increased by 1.44 times. Meanwhile, the MDA level of the ZEA + PD treatment group decreased by 1.92 times relative to the ZEA group. The T-SOD content of cells exposed to ZEA was significantly lower than that of the control group $(p<0.01$, Figure $3 \mathrm{~b})$. However, compared with ZEA treatment alone, the ZEA + PD treatment had significantly increased T-SOD content $(p<0.01)$. Finally, we evaluated the GSH content; we found that the GSH content of the ZEA treatment group was significantly lower than the control group $(p<0.001)$. Compared with the control group, the T-SOD activity of the ZEA treatment group decreased by 1.14 times. On the contrary, the GSH level of the ZEA + PD treatment group was significantly higher than that of the ZEA treatment group $(p<0.05)$. These results indicate that PD can inhibit the oxidative damage caused by ZEA in MAC-T cells. 


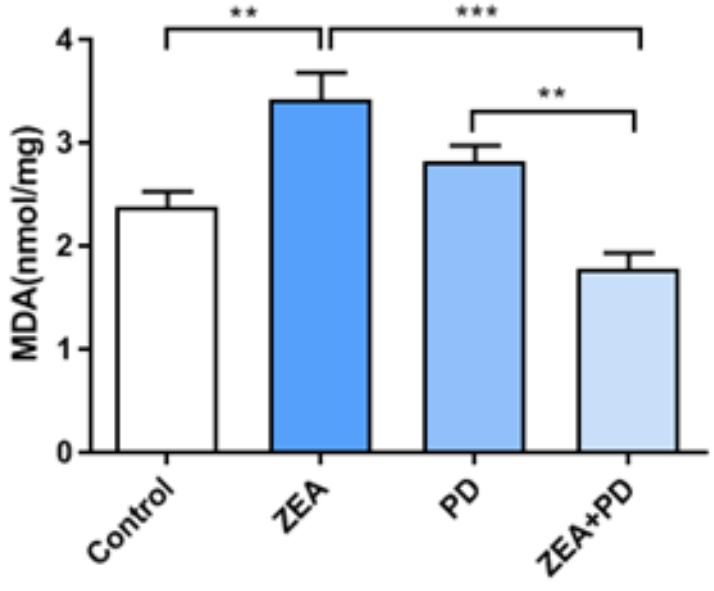

(a)

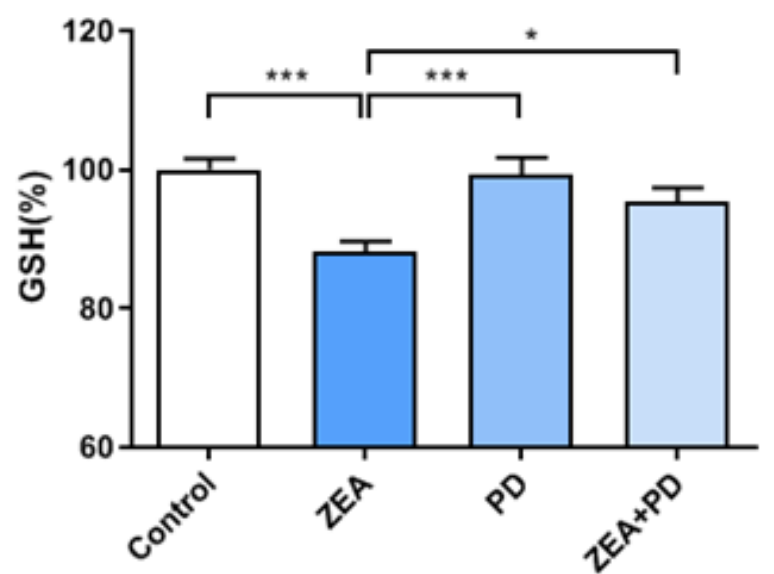

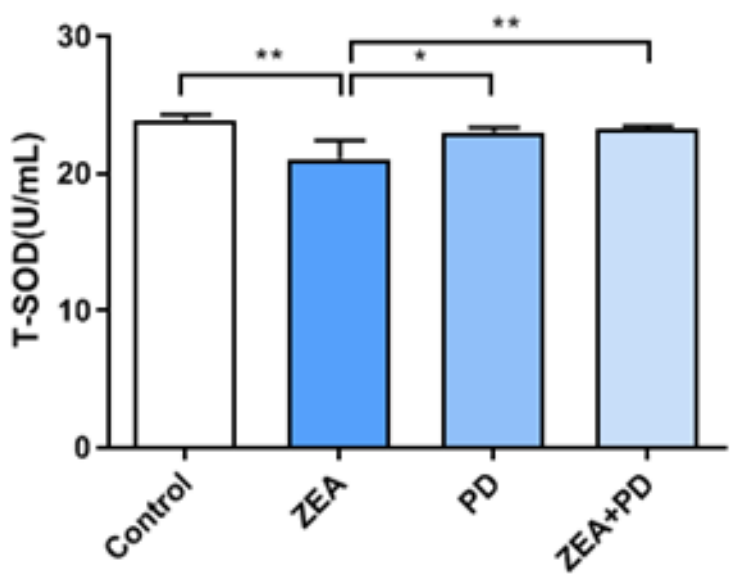

(b)

(c)

Figure 3. PD inhibits ZEA-induced oxidative damage in MAC-T cells. (a) MDA content, (b) T-SOD content, and (c) GSH content. All values are expressed as mean $\pm \operatorname{SEM}(n=3)$. In the figure, ${ }^{*}$ means $p<0.05,{ }^{* *}$ means $p<0.01$, and ${ }^{* * *}$ means $p<0.001$.

\subsection{PD Inhibits ZEA-Induced Increases in ROS Levels}

The above studies indicate that ZEA causes oxidative damage to MAC-T cells, and that PD can alleviate this situation. ROS mediates oxidative stress, and is a necessary marker for detecting ROS. The standard ROS detection system uses the fluorescent probe DCFH-DA. In this study, the ZEA treatment group had significantly higher active oxygen content than the control group $(p<0.001$; Figure $4 \mathrm{a}, \mathrm{b})$, and the ZEA + PD combination treatment significantly lowered the active oxygen content induced by the ZEA treatment $(p<0.001)$. While ZEA increased the ROS level by 2.94 times, adding PD reduced it by 1.97 times. 

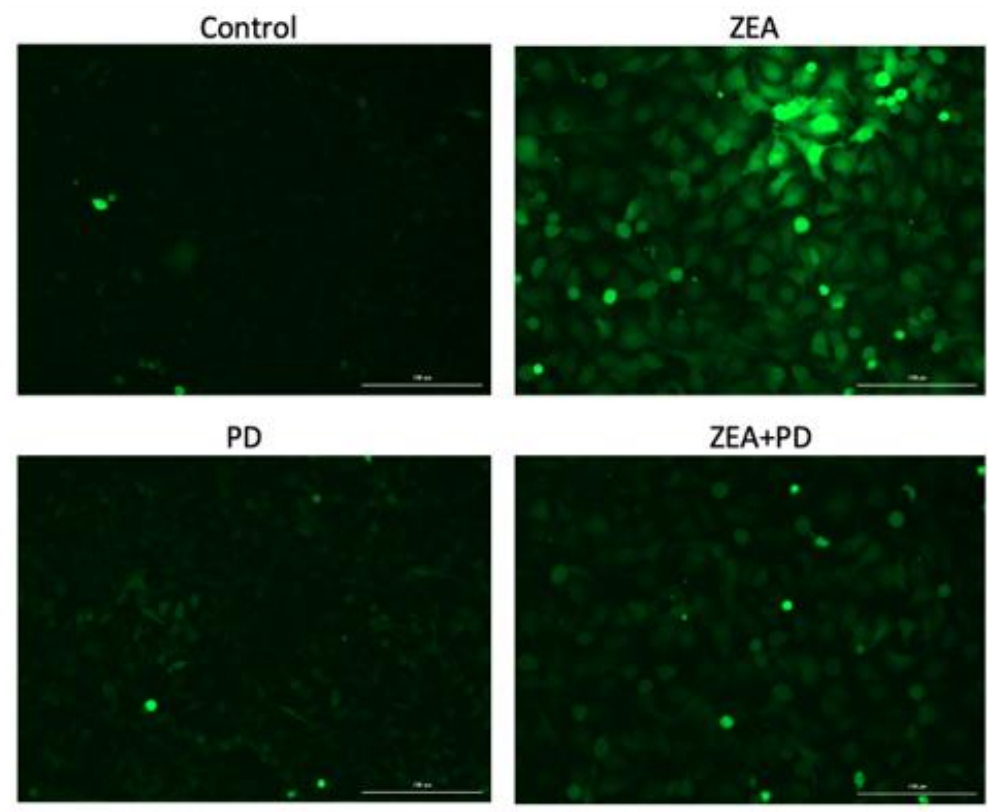

(a)

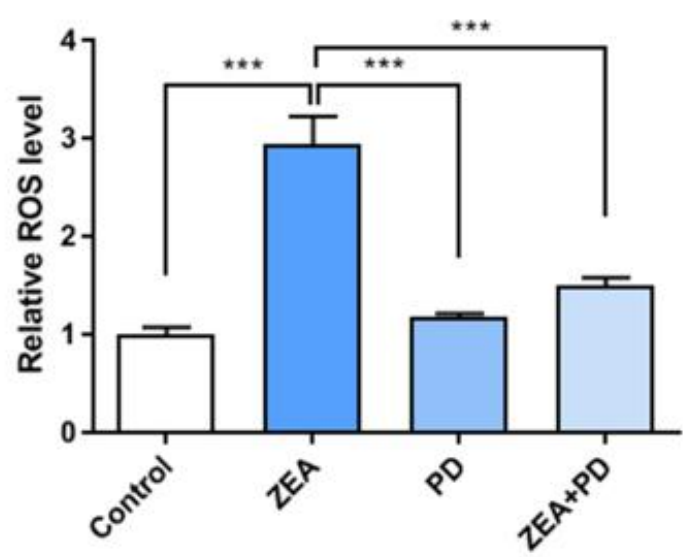

(b)

Figure 4. The effect of PD on ZEA-induced production of ROS in MAC-T cells. (a) MAC-T cells from the control, ZEA, PD, and ZEA + PD treatment groups stained with the DCFH-DA probe, and (b) their fluorescence intensity. In the figure, ${ }^{* * *}$ means $p<0.001$. Scale bar $=200 \mu \mathrm{M}$.

\subsection{PD Inhibits ZEA-Induced ER Stress in MAC-T Cells}

Both oxidative and ER stress participate in various physiological and pathophysiological conditions, and play a vital role in cell homeostasis and apoptosis. This study measured the expression levels of genes associated with ER stress to understand whether exposure to ZEA damages the ER function. The expressions of the ER stress markers GRP78 $(p<0.05)$, ATF4 $(p<0.01)$, ATF6 $(p<0.05)$, ASK1 $(p<0.05)$, and CHOP $(p<0.01)$ were significantly up-regulated in the ZEA treated group relative to the control group (Figure 5a-e). However, the expression levels of GRP78 $(p<0.05)$, ATF4 $(p<0.001)$, ATF6 $(p<0.05)$, and CHOP $(p<0.01)$ in the ZEA + PD treatment group were significantly lower than the ZEA treatment group. ASK1 expression showed a decreasing trend in the ZEA + PD group compared to the ZEA group. ZEA significantly increased the expressions of the ER stress marker genes by more than two-fold, but returned to normal levels after adding PD. 


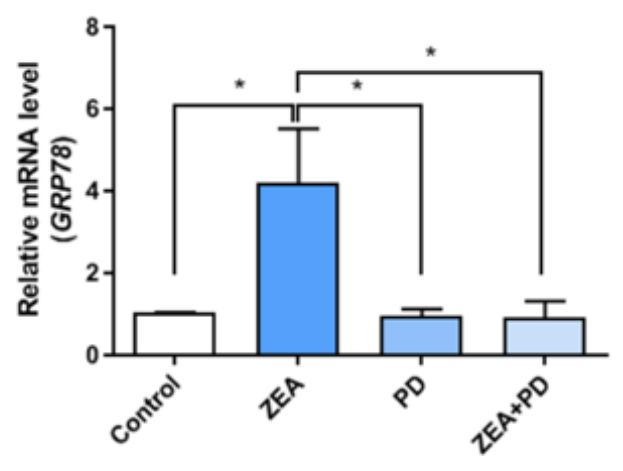

(a)

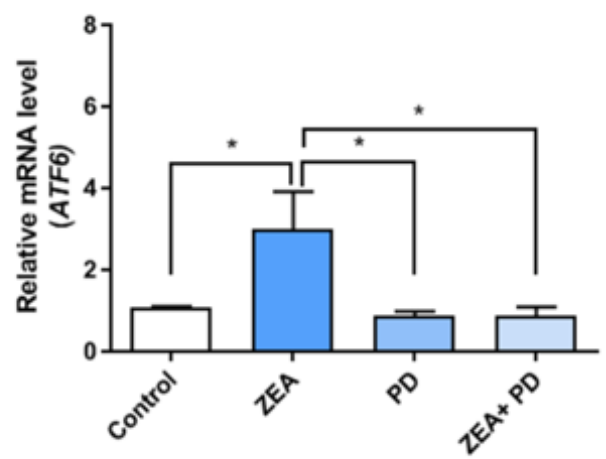

(c)

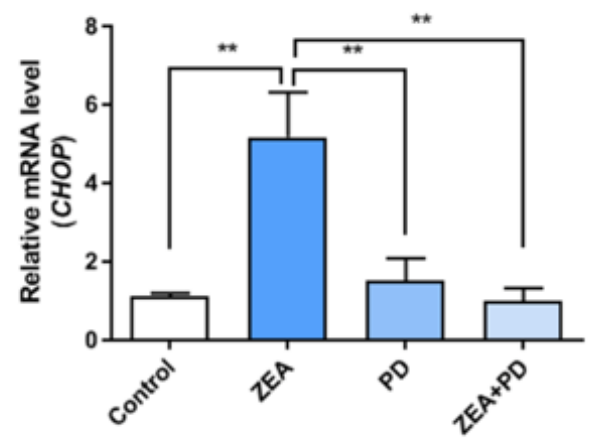

(e)

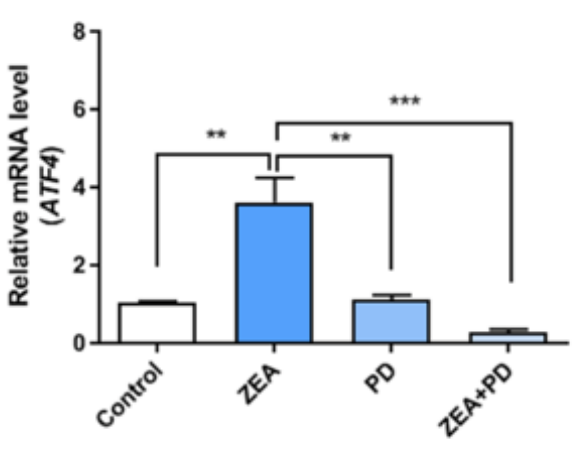

(b)

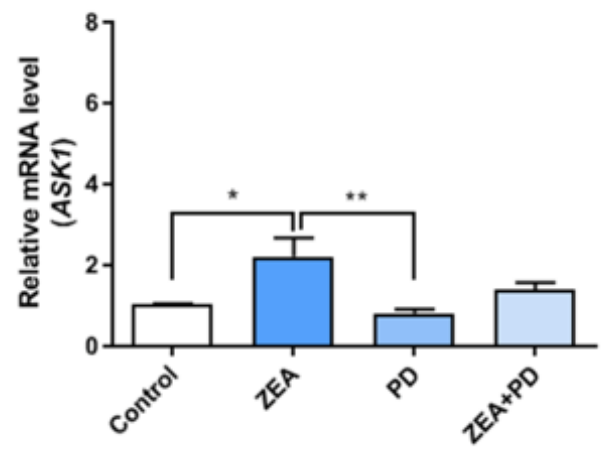

(d)

Figure 5. The effect of PD on the expressions of ER stress marker genes in MAC-T cells treated with by ZEA. (a-e) Analysis of gene expression levels of GRP78, ATF4, ATF6, ASK1, and CHOP. In the figure, ${ }^{*}$ means $p<0.05,{ }^{* *}$ means $p<0.01$, and ${ }^{* * *}$ means $p<0.001$.

\subsection{PD Inhibits ZEA-Induced Apoptosis}

Compared to the control, treating MAC-T cells with ZEA increased the apoptotic cell rate $(p<0.001$, Figure $6 \mathrm{a}, \mathrm{b})$; it increased from $10.22 \%$ to $29.67 \%$. The ZEA + PD treatment group had a significantly $(p<0.01)$ lower apoptotic rate $(29.67 \%)$ than the ZEA treatment group (29.06\%). Bax expression was significantly up-regulated $(p<0.01)$, while $B c l-2$ expression was significantly down-regulated $(p<0.05)$ in the ZEA-treated group relative to the control group (Figure $6 c, d$ ). In contrast, the ZEA + PD group had significantly lower Bax expression $(p<0.001)$ and significantly higher $B c l-2$ expression $(p<0.05)$ than ZEA alone. Finally, we measured the Caspase-3 activity using a Caspase-3 activity assay; the Caspase-3 activity of the ZEA-treated group was significantly higher than the control group $(p<0.05$, Figure 6e). However, the Caspase-3 activity of the ZEA + PD treatment group was significantly lower than ZEA alone $(p<0.01)$. Taken together, these results suggest that treating MAC-T cells with PD reduces the apoptotic effects of ZEA. 

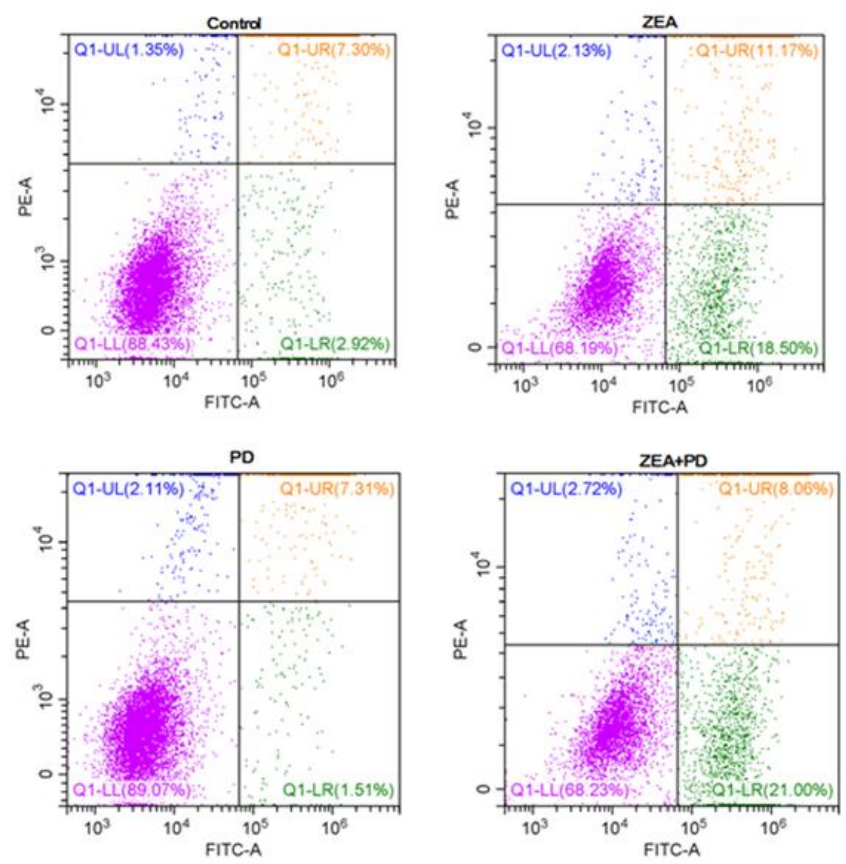

(a)

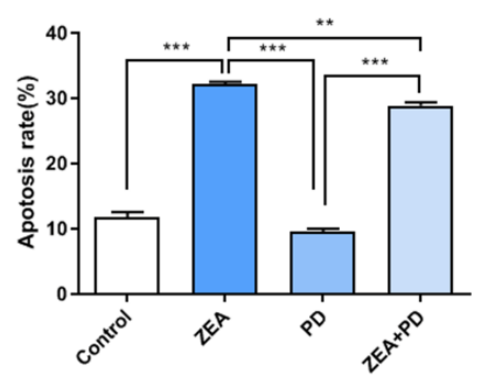

(b)

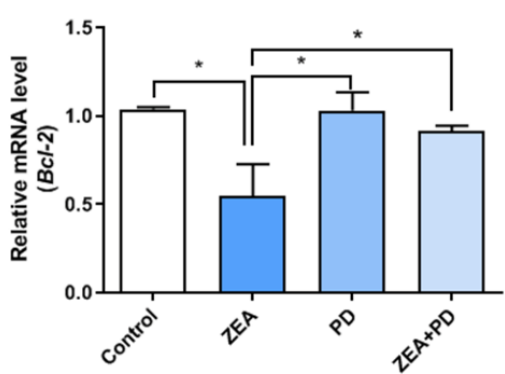

(d)

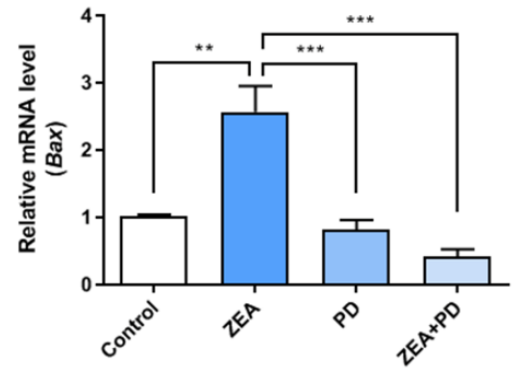

(c)

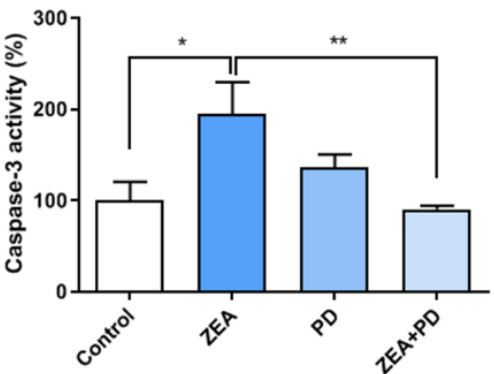

(e)

Figure 6. The effect of PD on ZEA-induced apoptosis in MAC-T cells. (a) Apoptosis measurements using annexin V/PI. The lower left panel contains annexin V and PI negative cells, while the lower right panel is annexin $\mathrm{V}$ positive. The upper left panel is annexin $\mathrm{V}$ - and $\mathrm{PI}+$, while the upper right panel is annexin $\mathrm{V}+$ and PI+. (b) Four groups of apoptotic rates. (c,d) qPCR analysis of mRNA expression levels of apoptosis-related genes Bcl-2 and Bax. (e) Four groups of Caspase-3 activity. In the figure, ${ }^{*}$ means $p<0.05,{ }^{* *}$ means $p<0.01$, and ${ }^{* * *}$ means $p<0.001$. 


\section{Discussion}

Feeding cows with a moldy feed containing mycotoxins results in a lower feed intake and reduced milk production [23]. In addition, the harmful mycotoxins in feed can be secreted in milk [24]. Currently, most countries have clear guidelines and detection limits for milk toxins. Although ZEA was detected in milk, the content may not be harmful to the human body. However, for the dairy cow itself, after feeding with the feed containing ZEA, if the ZEA is transferred to the milk through the bovine mammary gland, it cannot be ignored that ZEA is harmful. Therefore, it is necessary to evaluate the toxic effects of ZEA on breast cells at the cellular level and seek ways to protect breast cells from this damage, which is beneficial for the subsequent impact on milk production.

Zearalenone, a major toxin of animal feed, deserves attention. In our previous research, MAC-T cells were used as a model to study the effects of ZEA. It was found that ZEA can inhibit cell viability, reduce mitochondrial membrane potential, and cause ER stress and apoptosis [9]. In this study, PD, a protective agent, alleviated the effect of ZEA on bovine mammary epithelial cells. We found that ZEA decreased the viability of bovine mammary epithelial cells in a dose-dependent manner, consistent with our previous findings [9]. In another study, treating human hepatoma cells (HepG2 cells) with 50 to $250 \mu \mathrm{M}$ of ZEA for $24 \mathrm{~h}$ significantly reduced cell viability [25].

Recent pharmacological studies have shown that Polygonum cuspidatum has antibacterial, anti-inflammatory, diuretic, and other effects [26]. One of its main ingredients, PD, has various biological activities and pharmacological effects that are often cell type specific. Additionally, PD has anti-inflammatory and antioxidant properties, and can effectively treat health conditions and diseases [27]. Furthermore, PD is reported to improve arsenic damage to rat testicular cells [28] and inhibit the growth of some human tumor cells [29]. The cytotoxicity of a compound can be measured by the LDH levels released by cells [26]. Generally, an increase in LDH levels indicates increased cytotoxicity. However, adding PD significantly reduced LDH release by MAC-T cells exposed to ZEA.

SOD is an antioxidant enzyme, and its activity indirectly reflects the body's ability to eliminate free radicals. MDA is the end product of lipid peroxidation, and the MDA levels reflect the damage caused by lipid peroxidation [16]. Measuring the levels of these compounds can provide insights into the levels of cellular oxidative damage. ZEA treatment increased the MDA content and decreased GSH and T-SOD levels, suggesting that ZEA increases oxidative damage in cells. In another study, ZEA significantly increased the Glutathione peroxidase (GPx), Catalase (CAT), and SOD activities in the testis tissue of adult Balb/c male mice, but significantly increased the MDA in the same tissue [30]. However, all of the tested concentrations of $\mathrm{PD}$, including the lowest, alleviated the oxidative damage caused by ZEA on MAC-T cells. Exposing cells to both ZEA and PD reduced the MDA content and increased both GSH and T-SOD contents [28,31]. Studies have shown that PD can reduce oxidative damage in cardiomyocytes [19], consistent with the finding of the present study, where PD reduced ZEA-induced oxidative damage of MAC-T cells.

ER stress can activate mitochondrial pathways that trigger apoptosis, a reaction characterized by increased ROS production and lipid peroxidation, loss of mitochondrial transmembrane potential, activation of cysteine and acid proteases, and DNA damage [32,33]. We found that exposure to ZEA increased the ROS levels in MAC-T cells. It has previously been reported that ROS is produced in ZEA-treated leukemia cells (HL-60) [34]. However, treating cells with PD and ZEA reduced the ROS levels relative to the ZEA-treated group. Furthermore, PD can inhibit the damage induced by $S$. aureus lipoteichoic acid by attenuating ROS production [35]. These results concur with our findings that PD can effectively reduce the ROS contents of MAC-T cells.

GRP78 and CHOP are markers of ER stress [36], and the up-regulation of these genes indicates increased ER stress. We found that exposing MAC-T cells to ZEA significantly up-regulated these genes, as well as ATF4, ATF6, and ASK1. These results are consistent with previous studies [9]. However, treating ZEA-exposed cells with PD reduced the 
expression levels of GRP78, CHOP, ATF4, and ATF6 compared with the ZEA treatment group. These results show that PD can relieve the ZEA-induced ER stress on MAC-T cells.

The CCAAT/enhancer-binding protein homolog protein (CHOP) is a key pro-apoptotic transcription factor related to ER stress [32]. It is inadvertently known that the $B c l-2$ gene is anti-apoptotic, while the Bax gene is pro-apoptotic. CHOP-mediated ER stress inhibits $B c l-2$ expression, promoting Bax expression to induce apoptosis [37,38]. We found that cells exposed to ZEA had lower $\mathrm{Bcl}-2$ expression and elevated Bax expression. However, adding PD increased $B c l-2$ expression and decreased Bax expression. Flow cytometry evaluation of the apoptotic rate showed that ZEA treatment significantly increased apoptosis compared to the control group; however, the addition of PD significantly reduced the apoptosis rate. Although PD significantly reduced the cell apoptosis rate, the reduction degree was not large, indicating that $5 \mu \mathrm{M}$ of PD can ameliorate cell apoptosis, but not completely inhibit it. Banjerdpongchai et al. demonstrated that ZEA could activate Caspase- 3 activity in HL-60 and U937 cells in a dose-dependent manner [34]. In addition, Caspase-3 activity was significantly higher in ZEA-treated cells, and significantly decreased in the ZEA + PD treatment group compared to cells treated with ZEA. Our results are consistent with the anti-apoptotic properties of PD demonstrated by Liu et al. [39]. Taken together, our findings suggest that PD can attenuate ZEA-induced ER stress and apoptosis.

These results show that PD effectively reduces oxidative stress, ER stress, and apoptosis induced by ZEA; it may be a natural protective agent against ZEA. Therefore, this mechanism of PD should be studied further.

\section{Conclusions}

In vitro experiments revealed a new phenomenon that PD reduces ZEA-induced oxidative damage and ER stress in MAC-T cells by reducing ROS production, the activity of antioxidant enzymes, and the expression of ER stress-related genes. The stress subsequently alleviates cell apoptosis. These findings indicate that PD could be an effective antioxidant and potential therapeutic agent for diseases related to oxidative and ER stresses. However, the possible molecular mechanism of PD's protective effect on ZEA-induced cell damage in MAC-T cells needs further research. Thus, in vivo studies are required to determine the role of PD as an effective therapeutic agent that can be used as a feed additive.

\section{Materials and Methods}

\subsection{Chemicals and Reagents}

Zearalenone (ZEA, purity $>99 \%$ ), hydrocortisone, penicillin-streptomycin, and insulin were purchased from Sigma-Aldrich (St. Louis, MO, USA). ZEA was dissolved in dimethyl sulfoxide (DMSO, Sigma Chemical Co., St. Louis, MO, USA) and stored at $-20^{\circ} \mathrm{C}$. Polydatin (PD) was purchased from Refinsen Biotech Co., Ltd. (Chengdu, China), and dissolved in DMSO to generate a $100 \mathrm{mM}$ stock solution, which was stored at $-20{ }^{\circ} \mathrm{C}$. Fetal bovine serum (FBS) was purchased from Gibco (Gaithersburg, MD, USA), while Dulbecco's modified Eagle's/high-glucose medium (DMEM) was purchased from Hyclone (Logan, UT, USA). The cell counting kit-8 (CCK8) was procured from Dojindo Laboratories (Kumamoto, Japan), while the FITC Annexin V apoptosis detection kit was purchased from BD Biosciences (San Jose, CA, USA). Kits to measure ROS and caspase-3 activity were obtained from Beyotime Biotechnology (Shanghai, China). Kits for detecting the T-SOD, MDA, GSH, and lactate dehydrogenase (LDH) activities were purchased from Jiancheng Bioengineering Institute (Nanjing, China). Reagents for qPCR applications included SYBR green for real-time PCR (TransGen Biotech, Beijing, China) and the RevertAid First Strand cDNA Synthesis Kit (CW0581, Beijing, China).

\subsection{Cell Culture}

The bovine mammary epithelial cell line MAC-T was kindly provided by Professor Hong Gu Lee (Konkuk University, Seoul, Korea). For in vitro analyses, MAC-T cells were 
maintained in DMEM/high-glucose media containing 10\% FBS, $1 \%$ penicillin-streptomycin, $1 \mu \mathrm{g} / \mathrm{mL}$ of hydrocortisone, and $5 \mu \mathrm{g} / \mathrm{mL}$ of insulin, kept in a $37^{\circ} \mathrm{C}$ incubator with $5 \% \mathrm{CO}_{2}$.

\subsection{Cell Viability Assay}

Cell viability was measured using a CCK-8 kit (Dojindo Laboratories, Kumamoto, Japan) following the manufacturer's instructions. Briefly, MAC-T cells were seeded in 96-well plates at a density of $1 \times 10^{4}$ cells/well. When the cells reached 70-80\% confluence, they were treated with different concentrations of ZEA $(0,7.5,15,30,60,90,120$, and $240 \mu \mathrm{M})$ and $\mathrm{PD}(0,5,10$, and $20 \mu \mathrm{M})$ for $24 \mathrm{~h}$. A cell viability test was then performed; $10 \mu \mathrm{L}$ of CCK8 reagent were added to each well, and the cells were incubated for an additional $2.5 \mathrm{~h}$ at $37^{\circ} \mathrm{C}$, then randomly divided into four groups, each with three repeats. The experiments included the control, ZEA, PD, and ZEA + PD treatment groups. The absorbance at $450 \mathrm{~nm}$ was measured using a microplate reader (Eon, BioTek Instruments, USA), and cell viability was calculated as follows: (Treatment Group OD-Blank Group OD)/(Control Group OD-Blank Group OD).

\subsection{Lactate Dehydrogenase Assay}

We used an LDH kit (Nanjing Jiancheng Bioengineering Institute, Nanjing, China) to detect the LDH activity by the microplate method. The cells were plated in 96-well plates; ZEA and PD were treated when the cells' confluence reached 70-80\%. After $24 \mathrm{~h}$, the cell culture supernatant was collected for cytotoxicity level detection. Finally, the reagents were added according to the kit instructions, and the absorbance was measured with a microplate reader at a wavelength of $450 \mathrm{~nm}$.

\subsection{Detection of GSH, T-SOD, and MDA Levels}

The levels of GSH, T-SOD, and MDA were tested using the respective assay kits and following the manufacturer's protocol. As described above, MAC-T cells were treated with varying concentrations of ZEA and PD for $24 \mathrm{~h}$, and cells were collected to measure oxidation levels. The culture solution was also collected for later use. The cells were taken out with a cell scraper and transferred to a $1.5 \mathrm{~mL}$ centrifuge tube. Then, $500 \mu \mathrm{L}$ of the extract was added, and the contents were mixed by homogenization. Subsequently, $100 \mathrm{uL}$ of the mixture were transferred to another $1.5 \mathrm{~mL}$ centrifuge tube. The BCA kit determines the protein concentration. We measured the absorbance at $530 \mathrm{~nm}$ in the microplate reader. To determine the T-SOD content, the cells were cultivated in a similar way, then the cell protein concentration was evaluated. The kit instructions were used to add the reagents, which were mixed well and kept at room temperature for $10 \mathrm{~min}$, then the wavelength of $550 \mathrm{~nm}$ was colorimetrically detected. To determine the GSH content, cultured cells were taken out by cell scraping and transferred to a $1.5 \mathrm{~mL}$ centrifuge tube. A glass homogenizer was used for mixing; $100 \mu \mathrm{L}$ of the precipitation solution was taken and centrifuged at $3500 \mathrm{rpm}$ for $10 \mathrm{~min}$, and then the supernatant was taken for detection. The absorbance was measured at $405 \mathrm{~nm}$ in the microplate reader.

\subsection{Measurement of ROS Production}

The intracellular ROS levels of MAC-T cells were measured by a DCFH-DA kit (Shanghai, China). Cultures in multiple six-well plates were selected for the experiment. When the cell confluence reached $70-80 \%$, they were treated and cultured in a medium containing either ZEA or PD for $24 \mathrm{~h}$. Then, cultures were divided into control, ZEA, PD, and ZEA + PD treatment groups, and stained with $10 \mu \mathrm{L}$ of DCFH-DA for $30 \mathrm{~min}$ at $37^{\circ} \mathrm{C}$ in the dark. Cells were washed using $1 \times$ PBS to remove the unincorporated dye. The green fluorescence intensity was measured using the fluorescence microscope function of a Cytation five-cell imaging reader (BioTek Instruments, Winooski, VT, USA). Data were analyzed using the Gen5 3.03 software (BioTek Instruments, Winooski, VT, USA). 


\section{7. $q P C R$}

The total RNA was isolated using a TRIzol reagent (Invitrogen, Carlsbad, CA, USA). Complementary (cDNA) was synthesized from $1 \mu \mathrm{g}$ of RNA at $42^{\circ} \mathrm{C}$ for $15 \mathrm{~min}$, and then at $85^{\circ} \mathrm{C}$ for $5 \mathrm{~min}$. The SYBR Green Mix Kit was used to perform qPCR reactions. The qPCR reaction program was $94{ }^{\circ} \mathrm{C}$ for $30 \mathrm{~s}, 94{ }^{\circ} \mathrm{C}$ for $5 \mathrm{~s}, 60{ }^{\circ} \mathrm{C}$ for $15 \mathrm{~s}$, and $72{ }^{\circ} \mathrm{C}$ for $10 \mathrm{~s}$, respectively. A total of 40 cycles were performed. The mRNA levels were calculated by the $2^{-\Delta \Delta \mathrm{Ct}}$ comparative method and analyzed by normalization with $\beta$-actin mRNA expression. The primers used in the qPCR analyses are listed in Table 1.

Table 1. Gene name and PCR primer sequences.

\begin{tabular}{|c|c|c|c|c|}
\hline Gene & Forward Primer & Reverse Primer & $\begin{array}{c}\text { GenBank } \\
\text { Accession No. }\end{array}$ & $\begin{array}{c}\text { Product } \\
\text { Size (bp) }\end{array}$ \\
\hline$\beta$-actin & $5^{\prime}$-CCCTGGAGAAGAGCTACGAG-3' & 5'-GTAGTTTCGTGAATGCCGCAG-3' & nm_173979.3 & 130 \\
\hline GRP78 & 5'-CGACCCCTGACGAAAGACAA-3' & 5'-AGGTGTCAGGCGATTTTGGT-3' & nm_001075148.1 & 198 \\
\hline ATF4 & 5'-AGATGACCTGGAAACCATGC-3' & 5'-AGGGGGAAGAGGTTGAAAGA-3' & nm_001034342.2 & 190 \\
\hline ATF6 & 5'-ATATTCCTCCGCCTCCCTGT-3' & 5'-GTCCTTTCCACTTCGTGCCT-3' & XM_024989876.1 & 103 \\
\hline ASK1 & $5^{\prime}$-GCTATGGAAAGGCAGCCAGA-3' & $5^{\prime}$-TCTGCTGACATGGACTCTGG-3' & nm_001144081.2 & 160 \\
\hline CHOP & $5^{\prime}$-GAGCTGGAAGCCTGGTATGA-3' & $5^{\prime}$-CTCCTTGTTTCCAGGGGGTG-3' & nm_001078163.1 & 90 \\
\hline Bax & 5'-GCTCTGAGCAGATCATGAAGAC-3' & 5'-CAATTCATCTCCGATGCGCT-3' & nm_173894.1 & 167 \\
\hline Bcl-2 & 5'-GATGACCGAGTACCTGAACC-3' & $5^{\prime}$-AGAGACAGCCAGGAGAAATCA-3' & nm_001166486.1 & 123 \\
\hline
\end{tabular}

\subsection{Flow Cytometry Detection of Apoptosis}

Apoptosis was determined by staining cells with annexin $\mathrm{V}$ and propidium iodide (PI) using the Annexin V-FITC Apoptosis Detection Kit I (San Jose, CA, USA). MAC-T cells were first seeded in six-well plates and treated with ZEA and/or PD, as described above. After $24 \mathrm{~h}$, all of the cells and culture fluids were collected for subsequent experiments. At the time of sampling, we applied trypsin without EDTA to digest the cells, and then proceeded to the cell wash step. The cells were resuspended with pre-chilled $1 *$ PBS $\left(4{ }^{\circ} \mathrm{C}\right)$ and centrifuged at $1000 \mathrm{rpm}$ for $5 \mathrm{~min}$. This step was repeated thrice (trypsin was washed clean). After discarding the supernatants, cells were centrifuged, resuspended, and incubated for $15 \mathrm{~min}$ in $1 \times$ annexin binding buffer, $5 \mu \mathrm{L}$ of FITC Annexin V, and $5 \mu \mathrm{L}$ of PI working solutions. After incubation, cell apoptosis was detected by flow cytometry (Beckman-Coulter, Shanghai, China).

\subsection{Caspase-3 Activity Assay}

The caspase- 3 activity assay kit (Beyotime Biotechnology, Shanghai, China) was used to measure the caspase-3 activity levels following the manufacturer's protocols. Briefly, cells were trypsinized and centrifuged at $600 \times g$ for $5 \mathrm{~min}$. The supernatant was discarded, and the pellet was washed with $1 \times$ PBS and centrifuged at $600 \times g$ for $5 \mathrm{~min}$. The pellet was mixed with $100 \mu \mathrm{L}$ of lysate reagent to resuspend cells, and the mixture was incubated for $15 \mathrm{~min}$ on an ice bath. After $15 \mathrm{~min}$ of centrifugation at $16,000 \times \mathrm{g}$ and $4{ }^{\circ} \mathrm{C}$, the supernatant was transferred to a pre-chilled centrifuge tube, and the absorbance was measured at $405 \mathrm{~nm}$ using a microplate reader (BioTek Instruments, Winooski, VT, USA).

\subsection{Statistical Analyses}

The data were analyzed using the SPSS statistical software package. All experiments were repeated with three independent replicates. Statistical differences among the treatment groups were calculated using one-way ANOVA, and Duncan's Multiple Range Test was used for multiple comparisons. Data are expressed as mean \pm SEM. Differences of $p<0.05$ were considered statistically significant. 
Author Contributions: Conceptualization, Y.J. and J.Z.; Formal analysis, Y.Z.; Funding acquisition, J.Z.; Investigation, J.W. and Y.T.; Methodology, H.Y.; Project administration, A.S. and J.S.; Supervision, C.Z. and H.T.; Visualization, H.F. and H.Y.; Writing-original draft, Y.F.; Writing—review \& editing, Y.F. and Y.J. All authors have read and agreed to the published version of the manuscript.

Funding: This research was funded by the Scientific Research Project of Education Department of Jilin Province(JJKH20211133KJ), the Jilin Scientific and Technological Development Program (20190301034NY), P.R. China.

Institutional Review Board Statement: Not applicable.

Informed Consent Statement: Not applicable.

Data Availability Statement: Data sharing not applicable.

Acknowledgments: The MAC-T cell line was a gift from the laboratory of Professor Lee Hong-Gu, Konkuk University, Korea.

Conflicts of Interest: The authors declare that there is no conflict of interest with any commercial or financial organization regarding the material discussed in the manuscript.

\section{References}

1. Kouadio, J.H.; Mobio, T.A.; Baudrimont, I.; Moukha, S.; Dano, S.D.; Creppy, E.E. Comparative study of cytotoxicity and oxidative stress induced by deoxynivalenol, zearalenone or fumonisin B1 in human intestinal cell line Caco-2. Toxicology 2005, $213,56-65$. [CrossRef] [PubMed]

2. Tiemann, U.; Brussow, K.P.; Dannenberger, D.; Jonas, L.; Pohland, R.; Jager, K.; Danicke, S.; Hagemann, E. The effect of feeding a diet naturally contaminated with deoxynivalenol (DON) and zearalenone (ZON) on the spleen and liver of sow and fetus from day 35 to 70 of gestation. Toxicol. Lett. 2008, 179, 113-117. [CrossRef]

3. Liu, M.; Gao, R.; Meng, Q.; Zhang, Y.; Bi, C.; Shan, A. Toxic effects of maternal zearalenone exposure on intestinal oxidative stress, barrier function, immunological and morphological changes in rats. PLoS ONE 2014, 9, e106412. [CrossRef]

4. Yang, L.; Yang, W.; Feng, Q.; Huang, L.; Zhang, G.; Liu, F.; Jiang, S.; Yang, Z. Effects of purified zearalenone on selected immunological measurements of blood in post-weaning gilts. Anim. Nutr. 2016, 2, 142-148. [CrossRef]

5. Pietsch, C.; Noser, J.; Wettstein, F.E.; Burkhardt-Holm, P. Unraveling the mechanisms involved in zearalenone-mediated toxicity in permanent fish cell cultures. Toxicon Off. J. Int. Soc. Toxinology 2014, 88, 44-61. [CrossRef]

6. Sordillo, L.M.; Aitken, S.L. Impact of oxidative stress on the health and immune function of dairy cattle. Vet. Immunol. Immunopathol. 2009, 128, 104-109. [CrossRef]

7. Wang, C.C.; Chiang, Y.M.; Sung, S.C.; Hsu, Y.L.; Chang, J.K.; Kuo, P.L. Plumbagin induces cell cycle arrest and apoptosis through reactive oxygen species/c-Jun N-terminal kinase pathways in human melanoma A375.S2 cells. Cancer Lett. 2008, $259,82-98$. [CrossRef] [PubMed]

8. Moon, D.O.; Kim, M.O.; Kang, S.H.; Choi, Y.H.; Kim, G.Y. Sulforaphane suppresses TNF-alpha-mediated activation of NF-kappaB and induces apoptosis through activation of reactive oxygen species-dependent caspase-3. Cancer Lett. 2009, 274, 132-142. [CrossRef] [PubMed]

9. Fu, Y.R.; Jin, Y.C.; Zhao, Y.; Shan, A.; Fang, H.T.; Shen, J.L.; Zhou, C.H.; Yu, H.; Zhou, Y.F.; Wang, X.; et al. Zearalenone induces apoptosis inbovine mammary epithelial cells by activating endoplasmic reticulum stress. J. Dairy Sci. 2019, 102, 10543-10553. [CrossRef] [PubMed]

10. Santos, C.X.; Tanaka, L.Y.; Wosniak, J.; Laurindo, F.R. Mechanisms and implications of reactive oxygen species generation during the unfolded protein response: Roles of endoplasmic reticulum oxidoreductases, mitochondrial electron transport, and NADPH oxidase. Antioxid. Redox Signal. 2009, 11, 2409-2427. [CrossRef]

11. Samarghandian, S.; Azimi-Nezhad, M.; Farkhondeh, T.; Samini, F. Anti-oxidative effects of curcumin on immobilization-induced oxidative stress in rat brain, liver and kidney. Biomed. Pharmacother. 2017, 87, 223-229. [CrossRef]

12. Lin, P.; Chen, F.; Sun, J.; Zhou, J.; Wang, X.; Wang, N.; Li, X.; Zhang, Z.; Wang, A.; Jin, Y. Mycotoxin zearalenone induces apoptosis in mouse Leydig cells via an endoplasmic reticulum stress-dependent signalling pathway. Reprod. Toxicol. 2015, 52, 71-77. [CrossRef]

13. Kadowaki, H.; Nishitoh, H.; Ichijo, H. Survival and apoptosis signals in ER stress: The role of protein kinases. J. Chem. Neuroanat. 2004, 28, 93-100. [CrossRef]

14. Malhotra, J.D.; Kaufman, R.J. Endoplasmic reticulum stress and oxidative stress: A vicious cycle or a. double-edged sword? Antioxid. Redox Signal. 2007, 9, 2277-2293. [CrossRef]

15. Cao, S.S.; Kaufman, R.J. Endoplasmic reticulum stress and oxidative stress in cell fate decision and. human disease. Antioxid. Redox Signal. 2014, 21, 396-413. [CrossRef] 
16. Wang, H.L.; Gao, J.P.; Han, Y.L.; Xu, X.; Wu, R.; Gao, Y. Comparative studies of polydatin and resveratrol on mutual transformation and antioxidative effect in vivo. Phytomedicine 2015, 22, 553-559. [CrossRef] [PubMed]

17. Du, Q.H.; Peng, C.; Zhang, H. Polydatin: A review of pharmacology and pharmacokinetics. Pharm. Biol. 2013, 51, 1347-1354. [CrossRef]

18. Ji, H.; Zhang, X.; Du, Y.; Liu, H.; Li, S.; Li, L. Polydatin modulates inflammation by decreasing NF-kappaB activation and oxidative stress by increasing Gli1, Ptch1, SOD1 expression and ameliorates blood-brain barrier permeability for its neuroprotective effect in pMCAO rat brain. Brain Res. Bull. 2012, 87, 50-59. [CrossRef] [PubMed]

19. Jiang, X.; Liu, W.; Deng, J.; Lan, L.; Xue, X.; Zhang, C.; Cai, G.; Luo, X.; Liu, J. Polydatin protects cardiac function against burn injury by inhibiting sarcoplasmic reticulum $\mathrm{Ca}^{2+}$ leak by reducing oxidative modification of ryanodine receptors. Free Radic. Biol. Med. 2013, 60, 292-299. [CrossRef]

20. Ye, J.; Piao, H.; Jiang, J.; Jin, G.; Zheng, M.; Yang, J.; Jin, X.; Sun, T.; Choi, Y.H.; Li, L.; et al. Polydatin inhibits mast cell-mediated allergic inflammation by targeting PI3K/Akt, MAPK, NF-kappaB and Nrf2/HO-1 pathways. Sci. Rep. 2017, 7, 11895. [CrossRef] [PubMed]

21. Zhao, K.S.; Jin, C.; Huang, X.; Liu, J.; Yan, W.S.; Huang, Q.; Kan, W. The mechanism of Polydatin in shock treatment. Clin. Hemorheol. Microcirc. 2003, 29, 211-217. [CrossRef] [PubMed]

22. Chen, M.; Hou, Y.; Lin, D. Polydatin Protects Bone Marrow Stem Cells against Oxidative Injury: Involvement of Nrf 2/ARE Pathways. Stem Cells Int. 2016, 9394150. [CrossRef] [PubMed]

23. Bryden, W.L. Mycotoxin contamination of the feed supply chain: Implications for animal productivity and feed security. Anim. Feed Sci. Technol. 2012, 173, 134-158. [CrossRef]

24. Flores-Flores, M.E.; Lizarraga, E.; de Cerain, A.L.; González-Peñas, E. Presence of mycotoxins in animal milk: A review. Food Control 2015, 53, 163-176. [CrossRef]

25. Hassen, W.; Ayed-Boussema, I.; Oscoz, A.A.; Ade, C.L.; Bacha, H. The role of oxidative stress in zearalenone-mediated toxicity in Hep G2 cells: Oxidative DNA damage, gluthatione depletion and stress proteins induction. Toxicology 2007, 232, 294-302. [CrossRef]

26. Wu, M.; Liu, M.; Guo, G.; Zhang, W.; Liu, L. Polydatin Inhibits Formation of Macrophage-Derived Foam Cells. Evid.-Based Complementary Altern. Med. 2015, 729017. [CrossRef]

27. Koneru, M.; Sahu, B.D.; Gudem, S.; Kuncha, M.; Ravuri, H.G.; Kumar, J.M.; Kilari, E.K.; Sistla, R. Polydatin alleviates alcoholinduced acute liver injury in mice: Relevance of matrix metalloproteinases (MMPs) and hepatic anti-oxidants. Phytomedicine Int. J. Phytother. Phytopharm. 2017, 27, 23-32. [CrossRef]

28. Ince, S.; Avdatek, F.; Demirel, H.H.; Arslan-Acaroz, D.; Goksel, E.; Kucukkurt, I. Ameliorative effect of polydatin on oxidative stress-mediated testicular damage by chronic arsenic exposure in rats. Andrologia 2016, 48, 518-524. [CrossRef]

29. Liu, H.; Zhao, S.; Zhang, Y.; Wu, J.; Peng, H.; Fan, J.; Liao, J. Reactive oxygen species-mediated endoplasmic reticulum stress and mitochondrial dysfunction contribute to polydatin-induced apoptosis in human nasopharyngeal carcinoma CNE cells. J. Cell. Biochem. 2011, 112, 3695-3703. [CrossRef] [PubMed]

30. Ben Salah-Abbes, J.; Abbes, S.; Abdel-Wahhab, M.A.; Oueslati, R. Raphanus sativus extract protects against Zearalenone induced reproductive toxicity, oxidative stress and mutagenic alterations in male Balb/c mice. Toxicon Off. J. Int. Soc. Toxinology 2009, 53, 525-533. [CrossRef]

31. Li, R.P.; Wang, Z.Z.; Sun, M.X.; Hou, X.L.; Sun, Y.; Deng, Z.F.; Xiao, K. Polydatin protects learning and memory impairments in a rat model of vascular dementia. Phytomedicine Int. J. Phytother. Phytopharm. 2012, 19, 677-681. [CrossRef] [PubMed]

32. Quan, Z.; Gu, J.; Dong, P.; Lu, J.; Wu, X.; Wu, W.; Fei, X.; Li, S.; Wang, Y.; Wang, J.; et al. Reactive oxygen species-mediated endoplasmic reticulum stress and mitochondrial dysfunction contribute to cirsimaritin-induced apoptosis in human gallbladder carcinoma GBC-SD cells. Cancer Lett. 2010, 295, 252-259. [CrossRef]

33. Ben Salem, I.; Prola, A.; Boussabbeh, M.; Guilbert, A.; Bacha, H.; Abid-Essefi, S.; Lemaire, C. Crocin and Quercetin protect HCT116 and HEK293 cells from Zearalenone-induced apoptosis by reducing endoplasmic reticulum stress. Cell Stress Chaperones 2015, 20, 927-938. [CrossRef]

34. Banjerdpongchai, R.; Kongtawelert, P.; Khantamat, O.; Srisomsap, C.; Chokchaichamnankit, D.; Subhasitanont, P. Mitochondrial and endoplasmic reticulum stress pathways cooperate in zearalenone-induced apoptosis of human leukemic cells. J. Hematol. Oncol. 2010, 3, 50. [CrossRef] [PubMed]

35. Zhao, G.; Jiang, K.; Wu, H.; Qiu, C.; Deng, G.; Peng, X. Polydatin reduces Staphylococcus aureus lipoteichoic acid-induced injury by attenuating reactive oxygen species generation and TLR2-NFkappaB signalling. J. Cell. Mol. Med. 2017, 21, 2796-2808. [CrossRef] [PubMed]

36. Li, J.; Ni, M.; Lee, B.; Barron, E.; Hinton, D.R.; Lee, A.S. The unfolded protein response regulator GRP78/BiP is required for endoplasmic reticulum integrity and stress-induced autophagy in mammalian cells. Cell Death Differ. 2008, 15, 1460-1471. [CrossRef] [PubMed]

37. Dvash, E.; Har-Tal, M.; Barak, S.; Meir, O.; Rubinstein, M. Leukotriene C4 is the major trigger of stress-induced oxidative DNA damage. Nat. Commun. 2015, 6, 10112. [CrossRef] [PubMed] 
38. Chen, Q.-Q.; Yuan, A.-H.; Yang, J.; Bi-xiang, Z.H.A.; Zhang, M. Effect of acupuncture on the endoplasmic reticulum stress IRE1-CHOP pathway and the expression levels of Bax and Bcl-2 protein as well as genes in pancreatic tissue of rats with diabetes mellitus. World J. Acupunct. Moxibustion 2017, 27, 41-46. [CrossRef]

39. Liu, Y.H.; Huang, Q.H.; Wu, X.; Wu, J.Z.; Liang, J.L.; Lin, G.S.; Xu, L.Q.; Lai, X.P.; Su, Z.R.; Chen, J.N. Polydatin protects against acetaminophen-induced hepatotoxicity in mice via anti-oxidative and anti-apoptotic activities. Food Funct. 2018, 9, 5891-5902. [CrossRef] 